\title{
Quantification of Material Property Changes During Electrode Degradation in Polymer Electrolyte Fuel Cells Using X-ray Computed Tomography
}

\author{
Robin T. White ${ }^{1}$, Sebastian Eberhardt ${ }^{1}$, Marina Najm ${ }^{1}$, Francesco P. Orfino ${ }^{1}$, Monica Dutta ${ }^{2}$, and Erik \\ Kjeang ${ }^{1}$ \\ 1. Fuel Cell Research Laboratory (FCReL), School of Mechatronic Systems Engineering, Simon Fraser \\ University, Surrey BC, Canada. \\ 2. Ballard Power Systems, Burnaby BC, Canada
}

Recent developments in lab-based X-ray Computed Tomography (XCT) systems has provided the ability to obtain non-destructive 3-dimensional imaging in numerous research fields, such as geology, materials science, and biomedical imaging [1]. A unique and prevalent application of this technique is to acquire a set of topographies at different experimental stages to assess any dynamic changes and obtain 4-dimensional imaging of a system under study [2]. This methodology is well suited for the study of polymer electrolyte fuel cell systems where detailed imaging of the membrane electrode assembly (MEA) can be obtained while it is still assembled in its operational housing [ 3].

Polymer electrolyte fuel cells (PEFCs) are comprised of layered structures, each with their own material properties, providing the functionality to convert hydrogen and air into usable electrical power, heat, and water. These layers can be separated into: flow fields, gas diffusion layers, cathode/anode catalyst layers, and membrane. XCT imaging relies directly on the physical properties of materials, such as density and elemental composition, to provide absorption contrast within an imaging field-of-view, which allows for the clear identification of the various materials within PEFCs [4]. During the operational lifetime of a PEFC, significant changes to material properties in each of these layers can occur as a result of the significant stresses associated with operational swings of temperature, RH, and voltage. These changes can be detrimental to performance and lifetime; thus, it is imperative that a comprehensive understanding of the degradation mechanisms and their impact on PEFC material properties is achieved; then, suitable mitigation strategies can be implemented.

Previously, imaging of PEFC component degradation, particularly the cathode catalyst layer, which is vulnerable to damage during operational cycles, has primarily been limited to ex situ imaging, as well as, qualitative morphological changes $[5,6]$. Recent research by our group employing a Zeiss Versa $520 \mathrm{XCT}$ system with a custom device holder design has enabled the 4D in-situ visualization of the full MEA during degradation cycles [3,4]. Additional information that can still be gained relates to other fundamental material properties such as density and composition change, and visualizing these changes during 4D imaging.

In this work we present a novel approach using a 4D in-situ lab-based XCT imaging technique to obtain quantitative material changes that occur during an accelerated stress test of a cathode catalyst layer within an operational PEFC. Post data acquisition with image processing yields results of material property changes such as: density, platinum and ionomer loadings, crack formation and propagation, and thickness changes; highlighting the capabilities of this novel approach for lab-based XCT systems. Figure 1 shows the resultant structural changes following accelerated degradation cycling of a cathode catalyst layer where crack propagation and crack formation is clearly observed. Density and platinum fractional loss calculated from the obtained data set using a unique approach for quantification by labbased XCT is also highlighted [7]. 
References:

[1.] Merkle, A. \& Gelb, J., Recent Advancements in Laboratory X-ray Microscopes. Microsc. Microanal. 19, 1314-1315 (2013).

[2.] Gelb, J., Functionality to Failure: Materials Engineering in the 4th Dimension. Adv. Mat. Proc. 170, 14-18 (2012).

[3.] White, R. T., Wu, A., Orfino, F. P., Dutta, M. \& Kjeang, E., 4D in situ visualization of electrode degradation in fuel cells by X-ray computed tomography, presented at ECS PRiME 2016 Meeting Abstract MA2016-02 2519, Honolulu, HI, 2016.

[4.] White, R. T. et al., 3D Printed Flow Field and Fixture for Visualization of Water Distribution in Fuel Cells by X-ray Computed Tomography. J. Electrochem. Soc. 163, F1337-F1343 (2016).

[5.] Deevanhxay, P., Sasabe, T., Minami, K., Tsushima, S. \& Hirai, S., Oblique Soft X-Ray Tomography as a Non-Destructive Method for Morphology Diagnostics in Degradation of Proton-Exchange Membrane Fuel Cell. Electrochim. Acta 135, 68-76 (2014).

[6.] Hwang, G. S. et al., Phase-change-related degradation of catalyst layers in proton-exchangemembrane fuel cells. Electrochim. Acta 95, 29-37 (2013).

[7.] Funding for this research was provided by the Natural Sciences and Engineering Research Council of Canada, Canada Foundation for Innovation, British Columbia Knowledge Development Fund, and Ballard Power Systems through an Automotive Partnership Canada grant.

a)

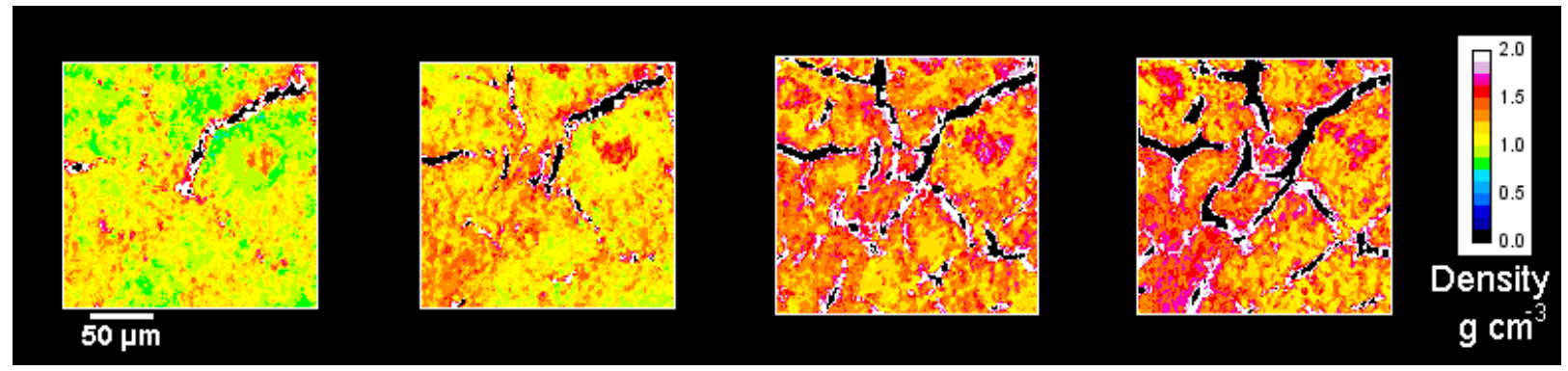

b)

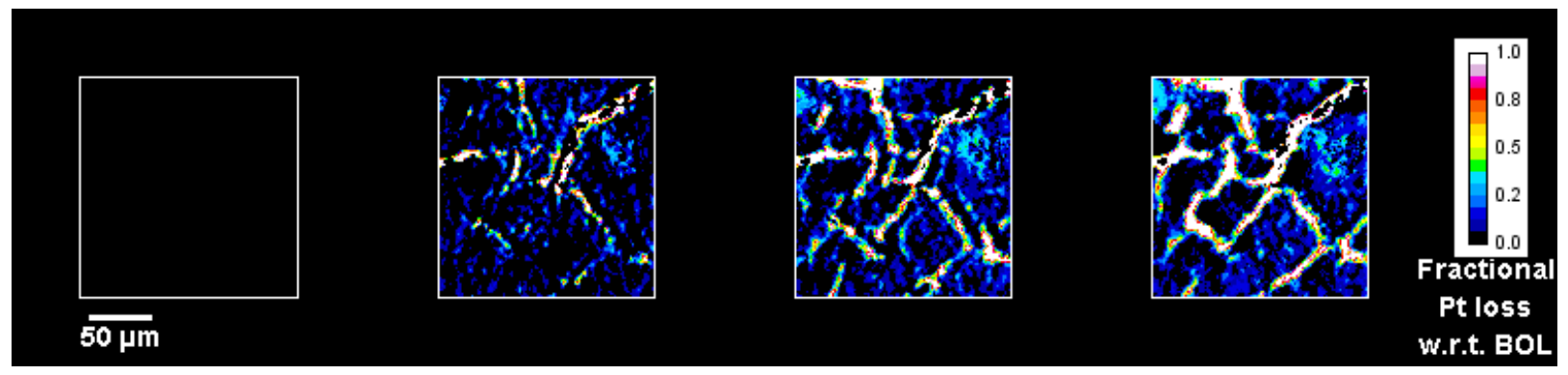

Increasing number of stress cycles

Figure 1. In-plane spatial distribution of (a) density and (b) fractional platinum loading loss with respect to beginning of life (BOL) following cathode catalyst degradation during accelerated stress test cycling obtained by quantitative XCT image analysis. It should be noted that the observed high density at the crack boundary may be a result of imaging resolution limitations and requires further investigation 